\title{
Measurement of the cosmic-ray secondary-to-primary ratios with CALET on the International Space Station
}

\author{
Yosui Akaike $^{a, b, *}$ and Paolo Maestro ${ }^{c, d}$ on behalf of the CALET Collaboration \\ (a complete list of authors can be found at the end of the proceedings) \\ ${ }^{a}$ Waseda Research Institute for Science and Engineering, Waseda University, 17 Kikuicho, Shinjuku-ku, \\ Tokyo 162-0044, Japan \\ ${ }^{b}$ JEM Utilization Center, Human Spaceflight Technology Directorate, Japan Aerospace Exploration \\ Agency, 2-1-1 Sengen, Tsukuba, Ibaraki 305-8508, Japan \\ ${ }^{c}$ Department of Physical Sciences, Earth and Environment, University of Siena, via Roma 56, 53100 \\ Siena, Italy \\ ${ }^{d}$ INFN Sezione di Pisa, Polo Fibonacci, Largo B. Pontecorvo, 3 - 56127 Pisa, Italy \\ E-mail: yakaike@aoni.waseda.jp
}

\begin{abstract}
The CALorimetric Electron Telescope, CALET, has been measuring high-energy cosmic rays on the International Space Station since October 2015. One of the scientific objectives of the CALET mission is the precise measurements of the energy spectra of individual cosmic-ray nuclei and the energy dependence of secondary-to-primary abundance ratio to reveal the detail of the cosmic-ray acceleration and propagation in the Galaxy. The instrument, consisting of two layers of segmented plastic scintillators, a 3 radiation length thick tungsten-scintillating fiber imaging calorimeter, and a 27 radiation length thick PWO calorimeter, has capabilities to identify individual nuclei elements up to $Z=40$ with excellent charge resolution and cover the wide energy range from $10 \mathrm{GeV}$ to a PeV scale. Long-term observation with CALET for over five years of operation allows to investigate the $\mathrm{TeV}$ region of the secondary components. In this paper, the details about the analysis of boron spectrum and boron-to-carbon ratio and their preliminary results will be presented.
\end{abstract}

$37^{\text {th }}$ International Cosmic Ray Conference (ICRC 2021)

July 12th - 23rd, 2021

Online - Berlin, Germany

\footnotetext{
*Presenter
} 


\section{Introduction}

It is commonly understood that the galactic cosmic rays are accelerated by diffusive shock acceleration in astrophysical sources like supernovae remnants, and diffusively propagate in the Galaxy. Since boron in the cosmic rays is produced in negligible quantities by nucleosynthesis processes in the star [1] but produced by the spallation of heavier nuclei such as carbon during the propagation in the interstellar medium, the boron-to-carbon $(\mathrm{B} / \mathrm{C})$ ratio is a direct probe to measure the average amount of interstellar material traversed by cosmic rays. In the context of the Leaky-Box approximation of particle transport in the Galaxy, the diffusion of cosmic rays is parameterized by introducing the average propagation path length, which is inversely proportional to the diffusion coefficient [2]. The energy dependence of the $\mathrm{B} / \mathrm{C}$ ratio can therefore provide the knowledge of the propagation in the Galaxy, and has been widely measured by balloon-borne and space-based experiments [3-9]. In addition, recently detailed measurements revealed a spectral hardening in proton, helium, and nucleus spectra at a few hundred $\mathrm{GeV} / n$ [10-18], and various theoretical models are proposed to explain the hardening (ex. [19-21]). The precise measurement of the $\mathrm{B} / \mathrm{C}$ ratio allows us to discriminate such models and to constrain the model parameters.

The CALorimetric Electron Telescope (CALET) operating on the International Space Station is measuring energy spectra of nuclei from proton to iron and above in the energy region above $10 \mathrm{GeV}$.The calorimeter is optimized for the measurement of electrons trans $\mathrm{TeV}$ region and has suitable features to measure nuclei. The detector has capabilities of charge identification with single element resolution and energy measurement to cover a wide energy range of 6 order of magnitude from $1 \mathrm{GeV}$ to $1 \mathrm{PeV}$. In this paper, we report the preliminary results of the energy spectrum of boron and boron-to-carbon ratio based on the data of five years of CALET operation.

\section{CALET Instrument}

The CALET instrument consists of three detectors; CHarge Detector (CHD), IMaging Calorimeter (IMC), and Total AbSorption Calorimeter (TASC). CHD and IMC play an important role in charge measurements and TASC for energy measurement. CHD located at the top part is composed of two layers of 14 plastic scintillator paddles for measurement of the primary particle charge from $Z=1-40$. Each scintillator has dimensions of $32 \mathrm{~mm} \times 450 \mathrm{~mm} \times 10 \mathrm{~mm}$. IMC for the track reconstruction and charge measurement is a sampling calorimeter composed of $(X, Y) \times 8$ layers of scintillating fiber ( $\mathrm{SciFi}$ ) belts and 7 tungsten plates. Each layer of SciFi belts is made of $448 \mathrm{SciFis}$ with a $1 \mathrm{~mm}$ square cross-section and $448 \mathrm{~mm}$ in length. The tungsten plates interleaved between the SciFi layers have a thickness of $0.2 X_{0} \times 5$ layers and $1.0 X_{0} \times 2$ layers from top to bottom. TASC is a total absorption calorimeter made of 12 layers of PWO scintillator logs for energy measurement and discrimination of electromagnetic shower and hadronic shower. Each layer has 16 PWO logs and each log has dimensions of $19 \mathrm{~mm} \times 326 \mathrm{~mm} \times 20 \mathrm{~mm}$. The total thickness of the calorimeter is $30 X_{0}$ for electromagnetic particles or $1.3 \lambda_{I}$ for protons. The detector performance is evaluated by MC simulations and several beam tests at CERN-SPS using electrons, protons and nuclei [22-24]. 


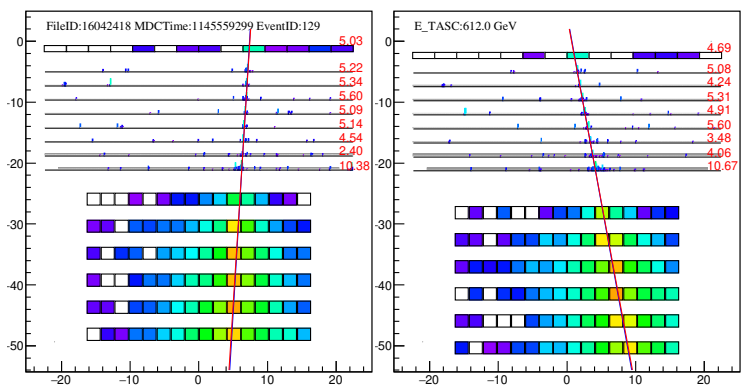

(a) $E_{T A S C}=612 \mathrm{GeV}$

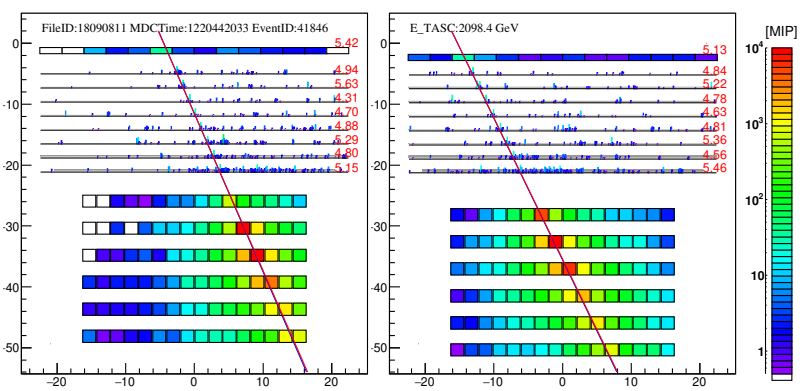

(b) $E_{T A S C}=2.10 \mathrm{TeV}$

Figure 1: Examples of boron candidates from the flight data. Red numbers show the reconstructed charges with each CHD and IMC layer.

\section{Data Analysis}

We have analyzed flight data collected over 1,815 days from October 13, 2015 to September 30, 2020. The total observation livetime is $T=3.69 \times 10^{4}$ hours. The field-of-view, FOV, of CALET within 45 degree is basically clear, while some structures of ISS and JEM-EF systems such as solar panels and JEM-RMS robotic arms partially shields the FOV. To avoid the contaminant for the measurement of the secondary cosmic rays, all events directed from such shielded region are discarded by the communication with ISS operation notes and manual inspection from the gamma-ray analysis [25]. Figure 1 shows the examples of boron events with $612 \mathrm{GeV}$ and $2.1 \mathrm{TeV}$ of observed energy. Monte Carlo simulation data are produced by EPICS v9.22 and Cosmos v8.02 [26] with DPMJET-III [27] as a hadron interaction model. The instrument configuration and detector response are detailed in the simulation code which provides digitized signals from all channels.

Nucleus events used in this analysis are detected by the high energy, HE, trigger [28] which requires a coincidence of two bottom layers of IMC and the top layer of TASC. The HE trigger mode always operates. The energy threshold is set to detect electrons above $10 \mathrm{GeV}$. Because the trigger threshold is higher than the $d E / d x$ of light nuclei such as boron and carbon, only events creating the particle shower in the detector are detected. Consistency between MC and FD for triggered events is obtained by an off-line trigger with higher threshold than the onbord trigger; 50 and 100 times a minimum ionizing particle (MIP) signals for IMC and TASC, respectively.

For the energy calibration of TASC, the laser irradiation test was carried out on the ground to check the linearity of the wide dynamic range from 1 MIP to $10^{6}$ MIP, which is corresponding to $1 \mathrm{PeV}$ shower energy. In addition, the minimum ionizing particles of cosmic-ray protons and helium detected in space are used for the calibration and monitoring of the detector performance including position, temperature, and time dependence of the plastic scintillators and PWO logs [23, 29]. For charge identification, the non-linearity of CHD and IMC between detector response and deposit energy caused by the scintillation quenching is corrected from the flight data [16].

The shower axis is reconstructed by IMC signals. Although the incident nuclei create shower particles in IMC as shown in Fig. 1, which could be a background for the track reconstruction, primary particle's signals are usually higher than the signals of the shower particles thanks to their 
large $d E / d x$. The shower axis is reconstructed by a least-square fit using the maximum energy channels in each upper four IMC layer. In addition, the different tracking procedures based on a combinational Kalman filter [30] are also used to study possible systematic uncertainties in tracking efficiency. The efficiency and accuracy between the two tracking methods are well consistent, and the systematic error by the tracking is negligibly small. Events with a fully reconstructed track are selected for the nuclei analysis in this paper, i.e. the track passes through the top surface of CHD and TASC and the bottom surface of TASC. The geometrical acceptance is $570.3 \mathrm{~cm}^{2}$ sr which is calculated using MC simulations.

Particle charge is identified based on the $d E / d x$ measurements in CHD and IMC associated with the reconstructed track. For the identification of the boron events, two selections are applied: the charge consistency among each CHD and IMC layer and the shower width at the initial shower stage in IMC. The charge consistency requires that the difference is less than $10 \%$ between CHD-X and $\mathrm{Y}$, and $15 \%$ between CHD and IMC. The charge consistency of 1st and 2nd IMC layers and 3rd and 4th layers are also required. The charge correlation between $Z_{\mathrm{IMC}}$ and $Z_{\mathrm{CHD}}$ is shown in Fig. 2 after applying only charge consistency of CHDs. Events with carbon undergoing a charge-changing nuclear interaction upstream the IMC are clearly visible in the tail of their drop-shaped distributions extending to lower $Z_{\mathrm{IMC}}$ value.

The major possible background is events interacting in CHD or upper surface materials. The shower width of such events at the initial shower stage is commonly wider than that of penetrating events due to the spread of the secondary particles, and the core signal of a nucleus passing through without an interaction shows larger signals than that of shower events. To exploit these characteristics, the difference of the sum signals in $7 \mathrm{SciFis}$ and the sum in $3 \mathrm{SciFis}$ normalized to the charge is used as the shower width parameters. Figure 3 shows the shower width distribution for boron candidates just applying the charge consistency cut with CHDs.

Figure 4 shows the charge distribution with CHD after applying charge consistency cuts and shower width selections. Particle charge is identified within \pm 0.4 charge unit in this analysis. The total efficiency is $20-25 \%$ above $E>20 \mathrm{GeV} / n$ as shown in Fig. 5. The isotopic composition of boron is assumed as ${ }^{11} \mathrm{~B} /\left({ }^{10} \mathrm{~B}+{ }^{11} \mathrm{~B}\right)=0.7$ for all energies [31, 32]. The assumptions with pure ${ }^{10} \mathrm{~B}$ and ${ }^{11} \mathrm{~B}$ make $1 \%$ differences in the efficiency, as shown in Fig. 5. The difference of the efficiency between the assumptions of pure ${ }^{10} \mathrm{~B}$ and ${ }^{11} \mathrm{~B}$ is $1 \%$ as shown in Fig. 5. The contamination from the other particles is estimated by MC events of elements with a charge in $Z=1-28$. The MC events are reweighted with a factor to reproduce a single power-law spectrum with the index of -2.60 and all events selections are identical to the ones used for the flight data. The absolute value of each element in each observed energy bin is normalized to the charge distribution of CHD matching the flight data. The number of contaminant events is calculated by the integration of all contamination MC events. The total background is less than $4 \%$ for boron as shown in Fig. 6, and the main background source is the carbon, which is increasing at higher energy because the abundance ratio of carbon respect to the boron is increasing at higher energy.

The shower energy for each event is determined by the sum of energy deposits in the TASC. Since the energy leakage from the calorimeter is unavoidable for nuclei due to the characteristics of hadron induced showers and finite detector resolution, an unfolding procedure is applied to derive the primary energy spectrum and to correct bin-to-bin migration. The iterative procedure based on Bayes's theorem [33] with the RooUnfold package [34] is applied with the response matrix of 

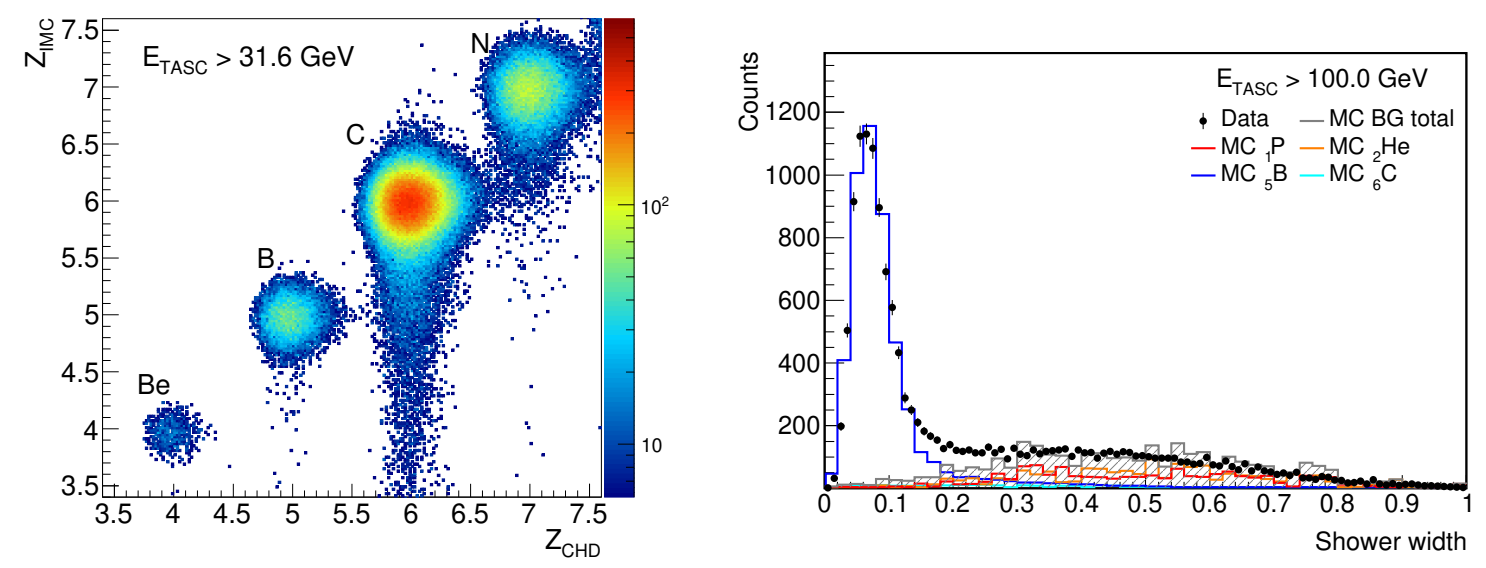

Figure 2: Crossplot of IMC versus CHD recon- Figure 3: The track width distribution compared with MC structed charges in the elemental range between reconstructed charges with $E_{\mathrm{TASC}}>100 \mathrm{GeV}$ Be and $\mathrm{O}$ with $E_{\text {TASC }}>31.6 \mathrm{GeV}$

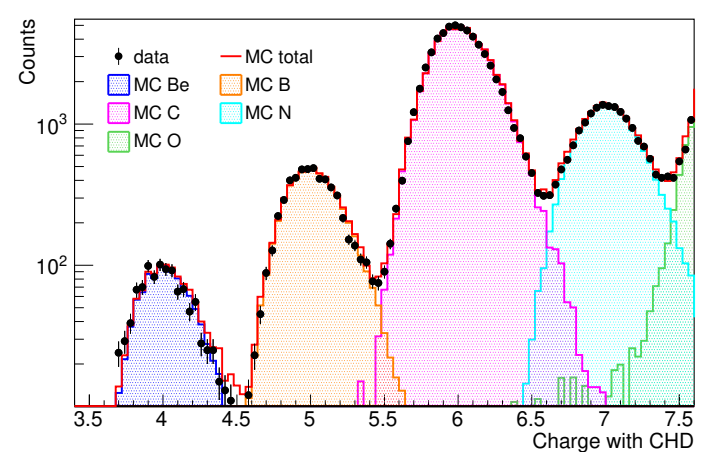

Figure 4: Charge distribution with $\mathrm{CHD}$ in $100<$ $E_{\mathrm{TASC}} /(\mathrm{GeV})<215$ compared with MC simulations

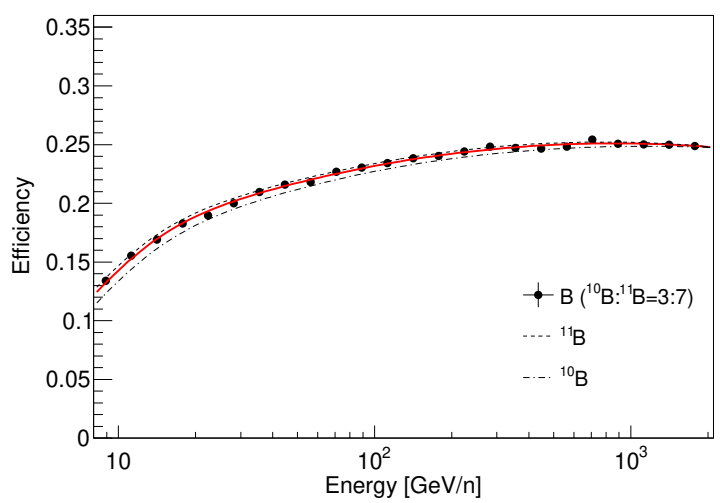

Figure 5: The total efficiency of boron

primary energy versus deposit energy, which is obtained from detailed MC simulation as shown in Fig. 7. The same event selections for MC data are applied as for flight data. Initial spectra are assumed as a single power-law function with the index of -2.60 and two cycles of unfolding iteration are applied.

\section{Results}

Energy flux, $\Phi(E)$, is calculated as follows,

$$
\begin{gathered}
\Phi(E)=\frac{N(E)}{\varepsilon S \Omega T \Delta E}, \\
N(E)=U\left[N_{\mathrm{obs}}\left(E_{\mathrm{TASC}}\right)-N_{\mathrm{bg}}\left(E_{\mathrm{TASC}}\right)\right],
\end{gathered}
$$

where $\Delta E$ denotes energy bin width, $E$ the particle kinetic energy, $N(E)$ is the bin content in the unfolded distribution, $\varepsilon(E)$ the total efficiency, $S \Omega$ the geometrical acceptance, $T$ the livetime, $U()$ the unfolding procedure, $N_{\mathrm{obs}}\left(E_{\mathrm{TASC}}\right)$ the bin content of observed energy distribution, $N_{\mathrm{bg}}\left(E_{\mathrm{TASC}}\right)$ 


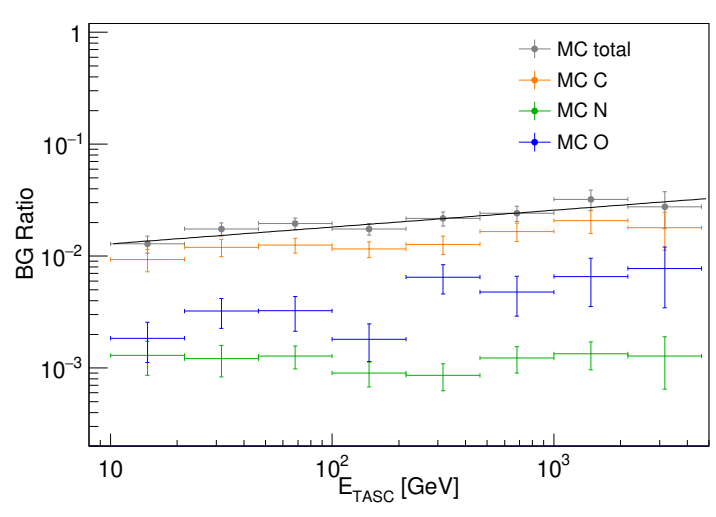

Figure 6: The fraction of the backgrounds for boron

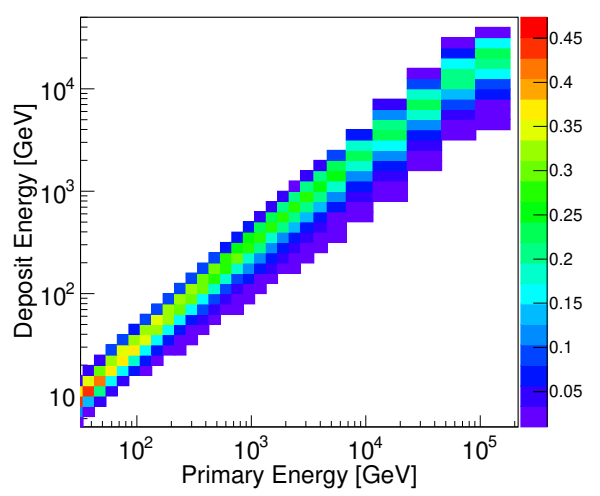

Figure 7: Response matrix for boron

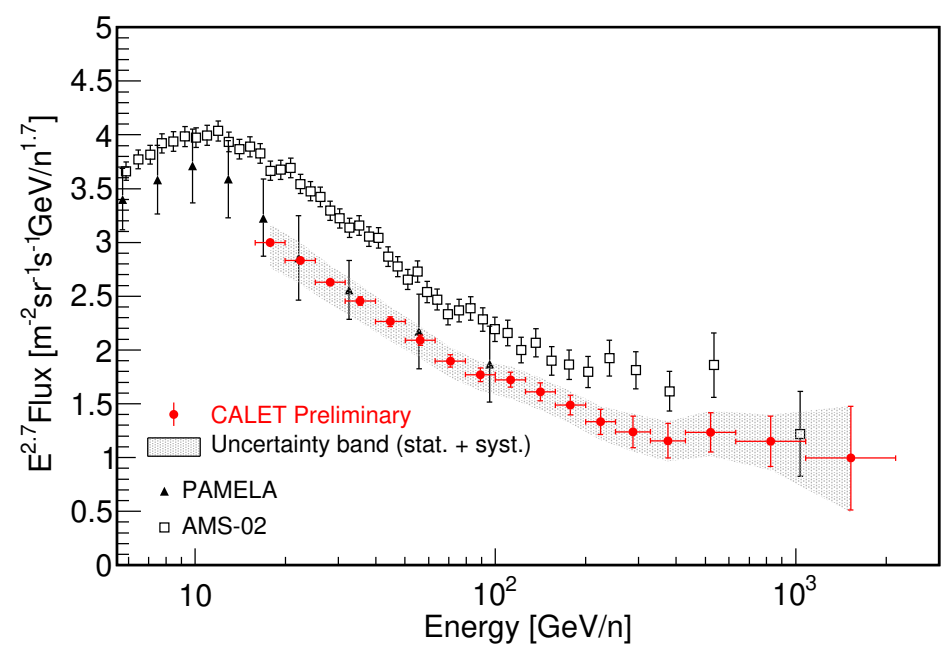

Figure 8: Preliminary energy spectrum of boron as a function of kinetic energy per nucleon with CALET compared with previous observations $[7,8]$.

the bin content of background events in the observed energy distribution. We have studied various systematic uncertainties such as trigger efficiency, charge identification, background estimation, energy unfolding, energy scale with beam test, MC model (EPICS/DPMJET-III and Geant4/FTFPBERT), live time, and long-term stability. Figure 8 shows the preliminary energy spectrum of boron as a function of kinetic energy per nucleon from $16 \mathrm{GeV} / n$ to $2.2 \mathrm{TeV} / n$, compared with PAMELA [7] and AMS-02 [8]. The isotope composition is assumed as ${ }^{11} \mathrm{~B} /\left({ }^{10} \mathrm{~B}+{ }^{11} \mathrm{~B}\right)=0.7$. The different assumptions of the isotope composition with ${ }^{11} \mathrm{~B} /\left({ }^{10} \mathrm{~B}+{ }^{11} \mathrm{~B}\right)=0.6$ and 0.8 make $2 \%$ differences in the boron spectrum. Our result is well consistent with PAMELA, but lower than AMS-02 like the cases of carbon, oxygen and iron spectra [16,35]. Figure 9 shows the preliminary result of $\mathrm{B} / \mathrm{C}$ ratio as a function of kinetic energy per nucleon from $16 \mathrm{GeV} / n$ to $2.2 \mathrm{TeV} / n$ compared with the previous observations [3-9]. Our present result of $\mathrm{B} / \mathrm{C}$ ratio can be fit with a power law function $(\mathrm{B} / \mathrm{C})=A E^{-\delta}$, where $\mathrm{A}$ is a constant normalization factor. The spectral indices are $\delta=0.406 \pm 0.039$ in $25 \mathrm{GeV} / n-100 \mathrm{GeV} / n$ with $\chi^{2} / n d f=0.30 / 3$, and $\delta=0.366 \pm 0.064$ in $100 \mathrm{GeV} / n-2.2 \mathrm{TeV} / n$ with $\chi^{2} / n d f=1.2 / 7$. 


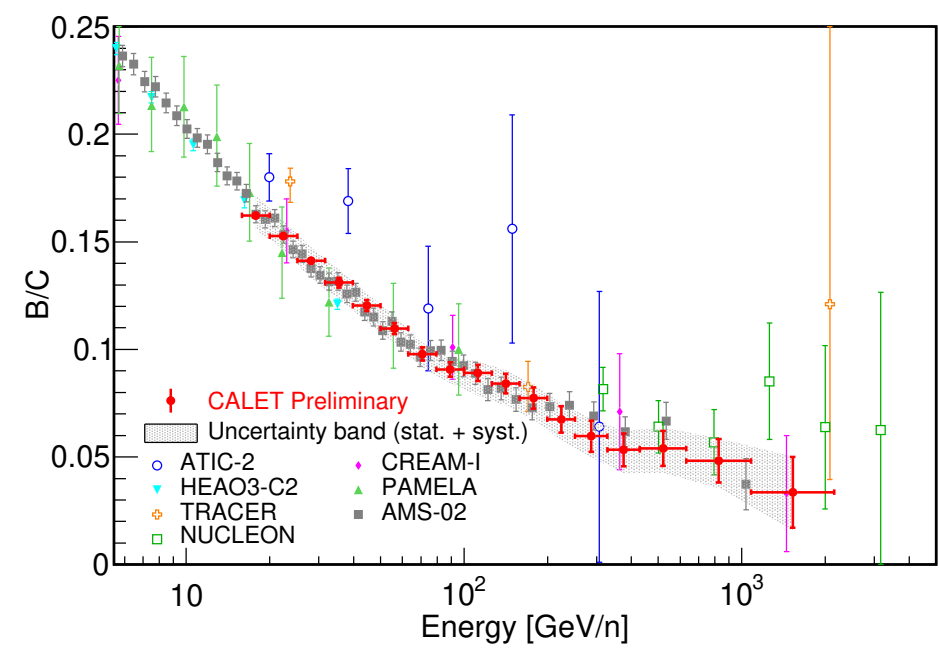

Figure 9: Preliminary result of the boron-to-carbon ratio as a function of kinetic energy with CALET compared with previous observations [3-9].

\section{Summary}

CALET on the ISS has measured the energy spectrum of boron and boron-to-carbon ratio from $16 \mathrm{GeV} / n$ to $2.2 \mathrm{TeV} / n$ after five years of operation. The preliminary spectrum of boron is in good agreement with PAMELA but lower than AMS-02. On the other hand, the B/C ratio is consistent with AMS-02 and the others, and the spectral indices below and above $100 \mathrm{GeV} / n$ are in agreement with AMS-02 within the errors[8]. CALET has capabilities to measure not only the $\mathrm{B} / \mathrm{C}$ ratio but also sub-Fe/Fe ratio, and the analysis is ongoing. Further studies on an increased data set and detailed systematic study will increase the sensitivity to detailed spectral features.

\section{Acknowledgement}

We gratefully acknowledge JAXA's contributions to the development of CALET and to the operations on the International Space Station. We also wish to express our sincere gratitude to ASI and NASA for their support of CALET project. This work was supported by JSPS KAKENHI Grant Numbers 20K22352 and 21K03592.

\section{References}

[1] H.A. Bethe, Phys. Rev. 55, 434 (1939)

[2] A.W. Strong, Annu. Rev. Nucl. 57, 285 (2007)

[3] J.J. Engelmann et al., Astron. Astrophys. 96, 233 (1990)

[4] A.D. Panov et al., Proc. of 30th ICRC 2, 3 (2008)

[5] H. Ahn et al., Astropart. Phys. 30, 133 (2008)

[6] A. Obermeier et al., Astropart. Phys. 752, 69 (2012) 
[7] O. Adriani et al., Astrophys. J. 791, 93 (2014)

[8] M. Aguilar et al., Phys. Rev. Lett. 120, 021101 (2018)

[9] V. Grebenyuk et al., Adv. Sp. Res. 64, 2559 (2019)

[10] O. Adriani et al., Science 322, 69 (2011)

[11] H. Ahn et al., Astrophys. J. 707, 14 (2011)

[12] M. Aguilar et al., Phys. Rev. Lett. 114, 171103 (2015)

[13] M. Aguilar et al., Phys. Rev. Lett. 115, 211101 (2015)

[14] M. Aguilar et al., Phys. Rev. Lett. 119, 251101 (2017)

[15] O. Adriani et al., Phys. Rev. Lett. 122, 181102 (2019)

[16] O. Adriani et al., Phys. Rev. Lett. 125, 251102 (2020)

[17] Q. An et al., Science Advances 5, No.9, eaax3793 (2019)

[18] F. Alemanno et al., Phys. Rev. Lett. 126, 201102 (2021)

[19] P. Blasi et al., Phys. Rev. Lett. 109, 061101 (2012)

[20] N. Tomassetti, Astrophys. J. Lett. 752, L13 (2012)

[21] Y. Ohira et al., Phys. Rev. D. 93, 083001 (2016)

[22] Y. Akaike et al., Proc. of 33rd ICRC, 0726 (2013)

[23] T. Niita et al., Adv. Sp. Res. 55, 9 (2015)

[24] Y. Akaike et al., PoS (ICRC2015) 613

[25] N. Cannady et al., in this proceedings

[26] K. Kasahara et al., Proc. of 24th ICRC 1, 399 (1995)

[27] S. Roesler et al., Proc. of Monte Carlo 71, 23 (2003)

[28] Y. Asaoka et al., Astropart. Phys. 100, 29 (2018)

[29] Y. Asaoka et al., Astropart. Phys. 91, 1 (2017)

[30] P. Maestro et al., PoS (ICRC2017 208 (2017))

[31] G. A. de Nolfo et al., AIP Conf. Proc. 598, 251 (2001)

[32] M. Aguilar et al., Phys. Rev. Lett. 117, 231102 (2016)

[33] G. D’Agostini et al., Nucl. Instrum. Meth. A 362, 487 (1995)

[34] T. Adye, arXiv:1105.1160 (2011)

[35] O. Adriani et al., Phys. Rev. Lett. 126, 241101 (2021) 


\section{Full Authors List: CALET Collaboration}

O. Adriani ${ }^{1,2}$, Y. Akaike ${ }^{3,4}$, K. Asano ${ }^{5}$, Y. Asaoka ${ }^{5}$, E. Berti ${ }^{1,2}$, G. Bigongiari 6,7 , W. R. Binns ${ }^{8}$, M. Bongi ${ }^{1,2}$, P. Brogi ${ }^{6,7}$, A. Bruno ${ }^{9,10}$, J. H. Buckley ${ }^{8}$, N. Cannady ${ }^{11,12,13}$, G. Castellini ${ }^{14}$, C. Checchia ${ }^{6}$, M. L. Cherry ${ }^{15}$, G. Collazuol ${ }^{16,17}$, K. Ebisawa ${ }^{18}$, A. W. Ficklin ${ }^{15}$, H. Fuke ${ }^{18}$, S. Gonzi ${ }^{1,2}$, T. G. Guzik ${ }^{15}$, T. Hams ${ }^{11}$, K. Hibino ${ }^{19}$, M. Ichimura ${ }^{20}$, K. Ioka $^{21}$, W. Ishizaki ${ }^{5}$, M. H. Israel ${ }^{8}$, K. Kasahara ${ }^{22}$, J. Kataoka ${ }^{23}$, R. Kataoka ${ }^{24}$, Y. Katayose ${ }^{25}$, C. Kato $^{26}$, N. Kawanaka ${ }^{27,28}$, Y. Kawakubo ${ }^{15}$, K. Kobayashi ${ }^{3,4}$, K. Kohri ${ }^{29}$, H. S. Krawczynski ${ }^{8}$, J. F. Krizmanic ${ }^{11,12,13}$,

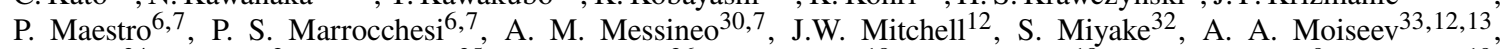
M. Mori ${ }^{34}$, N. Mori ${ }^{2}$, H. M. Motz ${ }^{35}$, K. Munakata ${ }^{26}$, S. Nakahira ${ }^{18}$, J. Nishimura ${ }^{18}$, G. A. de Nolfo ${ }^{9}$, S. Okuno ${ }^{19}$, J. F. Ormes $^{36}$, N. Ospina ${ }^{16,17}$, S. Ozawa ${ }^{37}$, L. Pacini ${ }^{1,14,2}$, P. Papini ${ }^{2}$, B. F. Rauch ${ }^{8}$, S. B. Ricciarini ${ }^{14,2}$, K. Sakai ${ }^{11,12,13 \text {, }}$ T. Sakamoto ${ }^{38}$, M. Sasaki ${ }^{33,12,13}$, Y. Shimizu ${ }^{19}$, A. Shiomi ${ }^{39}$, P. Spillantini ${ }^{1}$, F. Stolzi ${ }^{6,7}$, S. Sugita ${ }^{38}$, A. Sulaj ${ }^{6,7}$, M. Takita ${ }^{5}$, T. Tamura ${ }^{19}$, T. Terasawa ${ }^{40}$, S. Torii ${ }^{3}$, Y. Tsunesada ${ }^{41}$, Y. Uchihori ${ }^{42}$, E. Vannuccini ${ }^{2}$, J. P. Wefel ${ }^{15}$, K. Yamaoka ${ }^{43}$, S. Yanagita ${ }^{44}$, A. Yoshida ${ }^{38}$, K. Yoshida $^{22}$, and W. V. Zober ${ }^{8}$

${ }^{1}$ Department of Physics, University of Florence, Via Sansone, 1, 50019 Sesto, Fiorentino, Italy, ${ }^{2}$ INFN Sezione di Florence, Via Sansone, 1, 50019 Sesto, Fiorentino, Italy, ${ }^{3}$ Waseda Research Institute for Science and Engineering, Waseda University, 17 Kikuicho, Shinjuku, Tokyo 162-0044, Japan, ${ }^{4}$ JEM Utilization Center, Human Spaceflight Technology Directorate, Japan Aerospace Exploration Agency, 2-1-1 Sengen, Tsukuba, Ibaraki 305-8505, Japan, ${ }^{5}$ Institute for Cosmic Ray Research, The University of Tokyo, 5-1-5 Kashiwa-no-Ha, Kashiwa, Chiba 277-8582, Japan, ${ }^{6}$ Department of Physical Sciences, Earth and Environment, University of Siena, via Roma 56, 53100 Siena, Italy, ${ }^{7}$ INFN Sezione di Pisa, Polo Fibonacci, Largo B. Pontecorvo, 3, 56127 Pisa, Italy, ${ }^{8}$ Department of Physics and McDonnell Center for the Space Sciences, Washington University, One Brookings Drive, St. Louis, Missouri 63130-4899, USA, ${ }^{9}$ Heliospheric Physics Laboratory, NASA/GSFC, Greenbelt, Maryland 20771, USA, ${ }^{10}$ Department of Physics, Catholic University of America, Washington, DC 20064, USA, ${ }^{11}$ Center for Space Sciences and Technology, University of Maryland, Baltimore County, 1000 Hilltop Circle, Baltimore, Maryland 21250, USA, ${ }^{12}$ Astroparticle Physics Laboratory, NASA/GSFC, Greenbelt, Maryland 20771, USA, ${ }^{13}$ Center for Research and Exploration in Space Sciences and Technology, NASA/GSFC, Greenbelt, Maryland 20771, USA, ${ }^{14}$ Institute of Applied Physics (IFAC), National Research Council (CNR), Via Madonna del Piano, 10, 50019 Sesto, Fiorentino, Italy, ${ }^{15}$ Department of Physics and Astronomy, Louisiana State University, 202 Nicholson Hall, Baton Rouge, Louisiana 70803, USA, ${ }^{16}$ Department of Physics and Astronomy, University of Padova, Via Marzolo, 8, 35131 Padova, Italy, ${ }^{17}$ INFN Sezione di Padova, Via Marzolo, 8, 35131 Padova, Italy, ${ }^{18}$ Institute of Space and Astronautical Science, Japan Aerospace Exploration Agency, 3-1-1 Yoshinodai, Chuo, Sagamihara, Kanagawa 252-5210, Japan, ${ }^{19}$ Kanagawa University, 3-27-1 Rokkakubashi, Kanagawa, Yokohama, Kanagawa 221-8686, Japan, ${ }^{20}$ Faculty of Science and Technology, Graduate School of Science and Technology,, Hirosaki University, 3, Bunkyo, Hirosaki, Aomori 036-8561, Japan, ${ }^{21}$ Yukawa Institute for Theoretical Physics, Kyoto University, Kitashirakawa Oiwakecho, Sakyo, Kyoto 606-8502, Japan, ${ }^{22}$ Department of Electronic Information Systems, Shibaura Institute of Technology, 307 Fukasaku, Minuma, Saitama 337-8570, Japan, ${ }^{23}$ School of Advanced Science and Engineering, Waseda University, 3-4-1 Okubo, Shinjuku, Tokyo 169-8555, Japan, ${ }^{24}$ National Institute of Polar Research, 10-3, Midori-cho, Tachikawa, Tokyo 190-8518, Japan, ${ }^{25}$ Faculty of Engineering, Division of Intelligent Systems Engineering, Yokohama National University, 79-5 Tokiwadai, Hodogaya, Yokohama 240-8501, Japan, ${ }^{26}$ Faculty of Science, Shinshu University, 3-1-1 Asahi, Matsumoto, Nagano 390-8621, Japan, ${ }^{27}$ Hakubi Center, Kyoto University, Yoshida Honmachi, Sakyo-ku, Kyoto 606-8501, Japan, ${ }^{28}$ Department of Astronomy, Graduate School of Science, Kyoto University, Kitashirakawa Oiwake-cho, Sakyo-ku, Kyoto 6068502, Japan, ${ }^{29}$ Institute of Particle and Nuclear Studies, High Energy Accelerator Research Organization, 1-1 Oho, Tsukuba, Ibaraki 305-0801, Japan, ${ }^{30}$ University of Pisa, Polo Fibonacci, Largo B. Pontecorvo, 3, 56127 Pisa, Italy, ${ }^{31}$ Astroparticle Physics Laboratory, NASA/GSFC, Greenbelt, Maryland 20771, USA, ${ }^{32}$ Department of Electrical and Electronic Systems Engineering, National Institute of Technology, Ibaraki College, 866 Nakane, Hitachinaka, Ibaraki 312-8508, Japan ${ }^{33}$ Department of Astronomy, University of Maryland, College Park, Maryland 20742, USA, ${ }^{34}$ Department of Physical Sciences, College of Science and Engineering, Ritsumeikan University, Shiga 525-8577, Japan, ${ }^{35}$ Faculty of Science and Engineering, Global Center for Science and Engineering, Waseda University, 3-4-1 Okubo, Shinjuku, Tokyo 169-8555, Japan, ${ }^{36}$ Department of Physics and Astronomy, University of Denver, Physics Building, Room 211, 2112 East Wesley Avenue, Denver, Colorado 80208-6900, USA, ${ }^{37}$ Quantum ICT Advanced Development Center, National Institute of Information and Communications Technology, 4-2-1 Nukui-Kitamachi, Koganei, Tokyo 184-8795, Japan, ${ }^{38}$ College of Science and Engineering, Department of Physics and Mathematics, Aoyama Gakuin University, 5-10-1 Fuchinobe, Chuo, Sagamihara, Kanagawa 252-5258, Japan, ${ }^{39}$ College of Industrial Technology, Nihon University, 1-2-1 Izumi, Narashino, Chiba 275-8575, Japan ${ }^{40}$ RIKEN, 2-1 Hirosawa, Wako, Saitama 351-0198, Japan, ${ }^{41}$ Division of Mathematics and Physics, Graduate School of Science, Osaka City University, 3-3-138 Sugimoto, Sumiyoshi, Osaka 558-8585, Japan, ${ }^{42}$ National Institutes for Quantum and Radiation Science and Technology, 4-9-1 Anagawa, Inage, Chiba 263-8555, Japan, ${ }^{43}$ Nagoya University, Furo, Chikusa, Nagoya 464-8601, Japan, ${ }^{44}$ College of Science, Ibaraki University, 2-1-1 Bunkyo, Mito, Ibaraki 310-8512, Japan 